\title{
Vector Deviation for Recognition of Radar Target Intention
}

\author{
Dong YAN ${ }^{1}$, De-cun BIAN, Jin-chang ZHAO and Shao-qing NIU \\ College of Mining Technology, Taiyuan University of Technology, Taiyuan \\ 030000, China \\ Email:yandong0073@link.tyut.edu.cn
}

Keywords: Target intention; Eigenvector; Standard vector; Vector deviation.

\begin{abstract}
Radar data were used to develop judgement rules for target intention by analysis for known targets. For the eigenvector with corresponding dimension established by the known target, the standard vector determined by the air target vector with the known intention was adopted as the judging criteria. Matlab software was used to calculate the vector deviation between target and standard vectors to define intention clusters, and predict target intention. The assessment of suspicious target intention using vector deviation was validated to be rapid and precise.
\end{abstract}

\section{Introduction}

The radar reconnaissance is carried out by accepting the radiation signal of the air target radar. Then the spatial position and the technical parameters of the target are obtained [1,2]. After a large number of parameters of the target are obtained, it is needed to arrange and analyze the parameters. The air target intention is judged in aid of the effective computing method. Accordingly, it can be more pointed to take measures to the target rapidly and effectively.

A mathematical model is required to rapidly analyze the radar information and evaluate the target intention. The vector deviation is proposed as the basic method to judge a suspicious targets intention.

Suppose a target has a number of details, as listed successively, in Table 1.

Table 1. Air target information

\begin{tabular}{|c|c|c|}
\hline Number & Physical quantity & Value \\
\hline 1 & Azimuth angle, $B(\mathrm{mil})$ & 2300 \\
\hline 2 & Distance, $D(\mathrm{~km})$ & 210 \\
\hline 3 & Velocity, $V(\mathrm{~m} / \mathrm{s})$ & 300 \\
\hline 4 & Course angle, $\Theta($ degrees $)$ & 310 \\
\hline 5 & Height, $H(\mathrm{~km})$ & 4.0 \\
\hline 6 & Radar cross section, $\Sigma\left(\mathrm{m}^{2}\right)$ & 1.2 \\
\hline
\end{tabular}

A six-dimensional vector, $\mathbf{e}=(\beta, \mathrm{D}, \mathrm{v}, \theta, \mathrm{H}, \sigma)$ composed of 6 physical quantities corresponding to the target. The magnitude has little practical significance to judge

\footnotetext{
* Dong Yan (1989- ), male, Doctor of Engineering, studying in Taiyuan University of Technology, main science research direction: Wave, Data analysis theory and Impact dynamics. Correspondence address: Yingze West Street No.79, Taiyuan,Shanxi,China. Zip code:030000.
} 
the similarity between any two vectors $[3,4]$. However, the angle between two vectors provides a suitable measure of similarity, with smaller angle implying higher similarity [5]. Since vectors have a common origin (the radar station), two useful similarity measures can be defined: (1) whether the angle between the vectors is close to 0 , (2) whether the vector lengths are similar.

Suppose $\boldsymbol{A}=\left(a_{1}, a_{2}, a_{3} \ldots a_{\mathrm{n}}\right)$ and $\boldsymbol{B}=\left(b_{1}, b_{2}, b_{3} \ldots b_{\mathrm{n}}\right)$ are two n-dimensional vectors with the same starting point, as shown diagrammatically in Fig. 1.

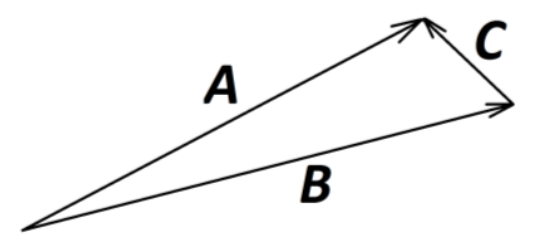

Figure 1. Schematic diagram of vector deviation, $\mathrm{C}$

When the vectors are close to equivalence, the angle between $\boldsymbol{A}$ and $\boldsymbol{B}$ is close to 0 , and the length of $\boldsymbol{C}$ is close to 0 . The vector deviation is defined as

$$
\omega(A, B)=\frac{|C|}{\min (|A|,|B|)}
$$

Matlab software programming ${ }^{[6,7]}$ :

function $\omega=\mathrm{f}(\mathrm{A}, \mathrm{B})$;

$\boldsymbol{A}=\left[a_{1}, a_{2}, a_{3} \ldots a_{\mathrm{n}}\right]$;

$\boldsymbol{B}=\left[b_{1}, b_{2}, b_{3} \ldots b_{\mathrm{n}}\right]$;

$\omega=\operatorname{sqrt}\left(\operatorname{sum}\left((\boldsymbol{A}-\boldsymbol{B}) .^{\wedge} 2\right)\right) /\left(\min \left(\operatorname{sqrt}\left(\operatorname{sum}\left(\boldsymbol{A} .^{\wedge} 2\right)\right), \operatorname{sqrt}\left(\operatorname{sum}\left(\boldsymbol{B} \cdot{ }^{\wedge} 2\right)\right)\right)\right) ;$

\section{Target Data}

The target data embody the information for judgment of intention. Targets are divided into two types: those with known intention and those with unknown and hence suspicious intention [8]. The data were derived from problem A in the Mathematical Contest in Modeling for graduate students, China in 2015, as listed in Table 2. 
Table 2. Targets with known intentions

\begin{tabular}{|c|c|c|c|c|c|c|c|c|}
\hline $\begin{array}{l}\text { Air } \\
\text { target }\end{array}$ & $\begin{array}{c}\text { Azimut } \\
\mathrm{h} \text { angel } \\
\beta(\mathrm{mil})\end{array}$ & $\begin{array}{c}\text { Distance } \\
D(\mathrm{~km})\end{array}$ & $\begin{array}{c}\text { Velocity } \\
v(\mathrm{~m} / \mathrm{s})\end{array}$ & $\begin{array}{c}\text { Course } \\
\text { angle } \\
\Theta\left(^{\circ}\right)\end{array}$ & $\begin{array}{c}\text { Heigh } \\
\mathrm{t} \\
H(\mathrm{~km})\end{array}$ & $\begin{array}{l}\text { Radar } \\
\text { cross } \\
\text { section } \\
\Sigma\left(\mathrm{m}^{2}\right)\end{array}$ & $\begin{array}{c}\text { Target } \\
\text { attribute }\end{array}$ & Intention \\
\hline 1 & 810 & 281 & 250 & 202 & 6.0 & 3.0 & $\begin{array}{c}\text { Medium } \\
\text { target }\end{array}$ & $\begin{array}{c}\text { Reconnai } \\
\text { ssance }\end{array}$ \\
\hline 2 & 2300 & 210 & 300 & 310 & 4.0 & 1.2 & $\begin{array}{l}\text { Small } \\
\text { target }\end{array}$ & Attack \\
\hline 3 & 820 & 280 & 245 & 201 & 6.5 & 5.4 & $\begin{array}{l}\text { Large } \\
\text { target }\end{array}$ & $\begin{array}{c}\text { Reconnai } \\
\text { ssance }\end{array}$ \\
\hline 4 & 2325 & 215 & 320 & 324 & 4.2 & 2.8 & $\begin{array}{l}\text { Medium } \\
\text { target }\end{array}$ & Attack \\
\hline 5 & 830 & 282 & 255 & 200 & 4.2 & 4.7 & $\begin{array}{l}\text { Large } \\
\text { target }\end{array}$ & $\begin{array}{c}\text { Reconnai } \\
\text { ssance }\end{array}$ \\
\hline 6 & 825 & 284 & 250 & 204 & 5.0 & 2.6 & $\begin{array}{c}\text { Medium } \\
\text { target }\end{array}$ & $\begin{array}{c}\text { Reconnai } \\
\text { ssance }\end{array}$ \\
\hline 7 & 2250 & 150 & 300 & 155 & 5.0 & 3.3 & $\begin{array}{c}\text { Medium } \\
\text { target }\end{array}$ & Attack \\
\hline 8 & 4000 & 110 & 300 & 50 & 3.4 & 2.1 & $\begin{array}{c}\text { Medium } \\
\text { target }\end{array}$ & Cover \\
\hline 9 & 2800 & 260 & 215 & 260 & 7.7 & 6.8 & $\begin{array}{l}\text { Large } \\
\text { target }\end{array}$ & $\begin{array}{c}\text { Surveilla } \\
\text { nce }\end{array}$ \\
\hline 10 & 5120 & 110 & 210 & 52 & 3.6 & 3.7 & $\begin{array}{l}\text { Medium } \\
\text { target }\end{array}$ & Other \\
\hline 11 & 4020 & 120 & 280 & 52 & 3.6 & 1.7 & $\begin{array}{l}\text { small } \\
\text { target }\end{array}$ & Cover \\
\hline 12 & 4800 & 140 & 220 & 18 & 9.6 & 5.7 & $\begin{array}{l}\text { Large } \\
\text { target }\end{array}$ & Other \\
\hline 13 & 480 & 295 & 292 & 245 & 9.9 & 6.9 & $\begin{array}{l}\text { Large } \\
\text { target }\end{array}$ & Other \\
\hline 14 & 2450 & 210 & 230 & 210 & 5.0 & 1.2 & $\begin{array}{l}\text { Small } \\
\text { target }\end{array}$ & Other \\
\hline 15 & 2900 & 290 & 272 & 350 & 5.6 & 5.2 & $\begin{array}{l}\text { Large } \\
\text { target }\end{array}$ & Attack \\
\hline
\end{tabular}

The vectors and intentions of known targets are shown in Table 3. The data also were derived from the Mathematical Contest in Modeling for graduate students. 
Table 3. Vectors and intentions of known targets

\begin{tabular}{|c|c|c|}
\hline $\begin{array}{c}\text { Known target } \\
\text { vector }\end{array}$ & Known target eigenvector & Intention \\
\hline$e_{1}$ & $(2300,210,300,310,4.0,1.2)$ & Attack \\
\hline$e_{2}$ & $(2325,215,320,324,4.2,2.8)$ & Attack \\
\hline$e_{3}$ & $(2250,150,300,155,5.0,3.3)$ & Attack \\
\hline$e_{4}$ & $(2900,290,272,350,5.6,5.2)$ & Attack \\
\hline$e_{5}$ & $(810,281,250,202,6.0,3.0)$ & Reconnaissance \\
\hline$e_{6}$ & $(820,280,245,201,6.5,5.4)$ & Reconnaissance \\
\hline$e_{7}$ & $(830,282,255,200,4.2,2.8)$ & Reconnaissance \\
\hline$e_{8}$ & $(825,284,250,204,5.0,2.6)$ & Reconnaissance \\
\hline$e_{9}$ & $(4000,110,300,50,3.4,2.1)$ & Cover \\
\hline$e_{10}$ & $(4020,120,280,52,3.6,1.7)$ & Cover \\
\hline$e_{11}$ & $(2800,260,215,260,7.7,6.8)$ & Surveillance \\
\hline$e_{12}$ & $(5120,110,210,52,3.6,3.7)$ & Other \\
\hline$e_{13}$ & $(4800,140,220,18,9.6,5.7)$ & Other \\
\hline$e_{14}$ & $(480,295,292,245,9.9,6.9)$ & Other \\
\hline$e_{15}$ & $(2450,210,230,210,5.0,1.2)$ & Other \\
\hline
\end{tabular}

Some physical information fed back by the target are transformed from the complex number into the uniform vector mode to realize the unified quantization of data. As above mentioned, 12 batches of the suspicious target data found by the radar are replaced by the vector. The eigenvectors for suspicious targets are obtained, as shown in Table 4. The data of suspicious target eigenvectors are the known conditions in Mathematical Contest in Modeling. 
Table 4. Suspicious target eigenvectors

\begin{tabular}{|c|c|c|c|}
\hline $\begin{array}{c}\text { ID of the } \\
\text { suspicious } \\
\text { target }\end{array}$ & Eigenvector & $\begin{array}{c}\text { ID of the } \\
\text { suspicious } \\
\text { target }\end{array}$ & Eigenvector \\
\hline 30 & $(2300,216,138,311,7,3.5)$ & 51 & $(1608,168,246,271,2.6,5.5)$ \\
\hline 31 & $(1279,211,225,253,9.2,5.7)$ & 60 & $(625,207,-126,217,9.4,6.2)$ \\
\hline 36 & $(1970,224,224,292,4.6,1.9)$ & 72 & $(2960,239,5.1,255,6,1.7)$ \\
\hline 37 & $(621,225,191,217,5.2,4.3)$ & 85 & $(2635,209,303,330,1.4,1.1)$ \\
\hline 39 & $(1250,233,95,252,5.2,5.5)$ & 91 & $(809,227,85,227,4.8,3.6)$ \\
\hline 42 & $(930,165,258,233,3.4,2.6)$ & 93 & $(1514,244,183,266,8.6,3.1)$ \\
\hline
\end{tabular}

Radar shows the flight path of suspicious targets. Fig.2 show the radar time and distance data for the suspicious targets of Table 4.
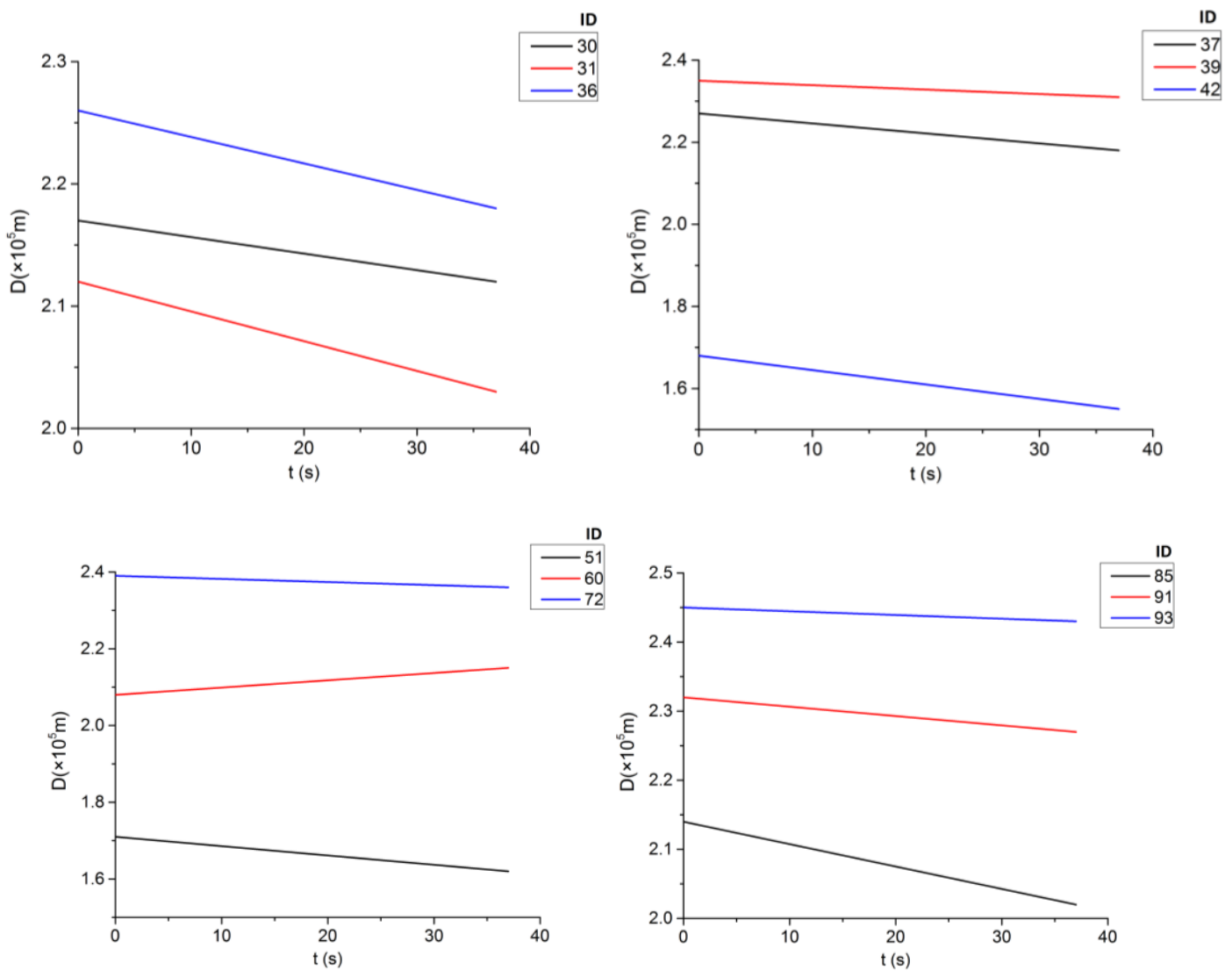

Figure 2. Radar time and distance of suspicious targets 30,31,36,37,39,42,51,60,72,85,91, and 93

\section{Relation Between Vector Deviation and Target Intention}

The vector deviation of known targets with the attack, reconnaissance, and cover intention are calculated. For the known targets with other intentions, such as civil, commercial transport, and agricultural aircraft, etc., it is difficult to accurately classify the target intention, and there is little practical significance. The known targets with other intention are independent of each other. Therefore, the eigenvectors in the other intentions are not the vectors of the same class of intentions. Tables 5-7 show the vector deviation for the attack, reconnaissance, and cover intention, respectively. The 
data of Tables 5-7 are supporting the results on vector recognition theory by the known conditions.

Table 5. Vector deviation for known targets with attack intention

\begin{tabular}{c|c|c}
\hline Known target vector & Intention & Vector deviation \\
\hline$e_{1}=(2300,210,300,310,4.0,1.2)$ & & $\omega\left(e_{1}, e_{2}\right)=0.0150$ \\
\hline$e_{2}=(2325,215,320,324,4.2,2.8)$ & & $\omega\left(e_{1}, e_{3}\right)=0.0761$ \\
\hline$e_{3}=(2250,150,300,155,5.0,3.3)$ & & $\omega\left(e_{1}, e_{4}\right)=0.2485$ \\
\hline$e_{4}=(2900,290,272,350,5.6,5.2)$ & & $\omega\left(e_{2}, e_{3}\right)=0.0864$ \\
& & $\omega\left(e_{2}, e_{4}\right)=0.1971$ \\
& & $\omega\left(e_{3}, e_{4}\right)=0.2795$ \\
\hline
\end{tabular}

Table 6. Vector deviation for known targets with reconnaissance intention

\begin{tabular}{c|l|l}
\hline \multicolumn{1}{c|}{ Known target vector } & $\begin{array}{l}\text { Intentio } \\
\mathrm{n}\end{array}$ & \multicolumn{1}{c}{ Vector deviation } \\
\hline$e_{5}=(810,281,250,202,6.0,3.0)$ & & $\omega\left(e_{5}, e_{6}\right)=0.0126$ \\
\hline$e_{6}=(820,280,245,201,6.5,5.4)$ & & $\omega\left(e_{5}, e_{7}\right)=0.0227$ \\
\hline$e_{7}=(830,282,255,200,4.2,2.8)$ & Reconna & $\omega\left(e_{5}, e_{8}\right)=0.0169$ \\
\cline { 1 - 1 }$=(825,284,250,204,5.0,2.6)$ & issance & $\omega\left(e_{6}, e_{7}\right)=0.0160$ \\
& & $\omega\left(e_{6}, e_{8}\right)=0.0100$ \\
& & $\omega\left(e_{7}, e_{8}\right)=0.0090$ \\
\hline
\end{tabular}

Table 7. Vector deviation for known targets with cover intention

\begin{tabular}{c|l|c}
\hline Known target vector & Intention & Vector deviation \\
\hline$e_{9}=(4000,110,300,50,3.4,2.1)$ & & \\
\hline$e_{10}=(4020,120,280,52,3.6,1.7)$ & & $\omega\left(e_{9}, e_{10}\right)=0.0075$ \\
\hline
\end{tabular}

The vector deviation between known targets with the same intention are distributed within 0.3 according to the Tables 5-7. Most deviations are very small, arguing that the vectors are similar. That is, the similarity of target vectors directly reflects the 
similarity of target intentions.

Therefore, it is verified that the similarity of the target vector reflects the closeness degree of the target intentions by checking the deviation degree of eigenvector of different intentions. Table 8 shows the vector deviation for known targets with different intentions. For space limitations, only indicative comparisons are shown.

Table 8. Vector deviation for known targets with different intentions

\begin{tabular}{|c|c|c|}
\hline Eigenvector of known target & Intention & Vector deviation \\
\hline$e_{1}=(2300,210,300,310,4.0,1.2)$ & Attack & $\omega\left(e_{1}, e_{6}\right)=1.6113$ \\
\hline$e_{6}=(820,280,245,201,6.5,5.4)$ & Reconnaissance & $\omega\left(e_{1}, e_{9}\right)=0.7332$ \\
\hline$e_{9}=(4000,110,300,50,3.4,2.1)$ & Cover & $\omega\left(e_{1}, e_{11}\right)=0.4360$ \\
\hline$e_{11}=(2800,260,215,260,7.7,6.8)$ & Surveillance & $\omega\left(e_{1}, e_{12}\right)=3.4559$ \\
\hline$e_{12}=(5120,110,210,52,3.6,3.7)$ & \multirow{4}{*}{ Other } & $\omega\left(e_{6}, e_{9}\right)=2.1473$ \\
\hline$e_{13}=(4800,140,220,18,9.6,5.7)$ & & $\omega\left(e_{6}, e_{11}\right)=0.4344$ \\
\hline$e_{14}=(480,295,292,245,9.9,6.9)$ & & $\omega\left(e_{6}, e_{13}\right)=1.2066$ \\
\hline$e_{15}=(2450,210,230,210,5.0,1.2)$ & & $\begin{array}{r}\omega\left(e_{9}, e_{11}\right)=4.6670 \\
\omega\left(e_{9}, e_{14}\right)=0.5600 \\
\omega\left(e_{11}, e_{15}\right)=0.8241\end{array}$ \\
\hline
\end{tabular}

Tables 5-8 show that the deviations of known target eigenvectors within the same intention class are much smaller than for targets with different intentions. The deviation of a known target within the same intention class is typically no more than 0.1 , with maximum less than 0.3. However, most deviations for different intentions are larger than 1 , with minimum approximately 0.43 . Therefore, the deviation between two target vectors reflects the closeness of target intentions.

\section{Judgement of Suspicious Target Intention}

\section{Vector Deviation of Suspicious Target}

The vector deviations are calculated for fifteen suspicious targets with known intention. In each intention class, the vector deviation between the suspicious targets within that class must be smaller than those of other classes. Then the vector can be seen as the baseline for the judgement of the class of intentions. It is referred to as the standard vector. Table 9 lists the standard vector of five classes of intentions. 
Table 9. Standard vector for predicting target intention

\begin{tabular}{c|c}
\hline Standard vector deviation & $\begin{array}{c}\text { Predicted } \\
\text { intension }\end{array}$ \\
\hline $\mathrm{e}_{2}=(2300,210,300,310,4.0,1.2)$ & Attack \\
\hline $\mathrm{e}_{3}=(820,280,245,201,6.5,5.4)$ & Reconnaissance \\
\hline $\mathrm{e}_{9}=(4000,110,300,50,3.4,2.1)$ & Cover \\
\hline $\mathrm{e}_{11}=(2800,260,215,260,7.7,6.8)$ & Surveillance \\
\hline $\mathrm{e}_{12}=(5120,110,210,52,3.6,3.7)$ & Other \\
\hline $\mathrm{e}_{13}=(4800,140,220,18,9.6,5.7)$ & Other \\
\hline $\mathrm{e}_{14}=(480,295,292,245,9.9,6.9)$ & Other \\
\hline $\mathrm{e}_{15}=(2450,210,230,210,5.0,1.2)$ & Other \\
\hline
\end{tabular}

Twelve suspicious targets are assessed for their intention by comparison to the standard vectors, as shown in Table 10. If the vector deviation between a standard vector and the suspicious target is very small, then the suspicious target intention may be assumed to be that of the standard vector. 
Table 10. Vector deviation between suspicious targets and standard vectors

\begin{tabular}{|c|c|c|c|c|c|c|c|c|}
\hline \multirow{2}{*}{$\begin{array}{l}\text { Suspicious } \\
\text { target } \\
\text { ID }\end{array}$} & \multicolumn{8}{|c|}{ Vector deviation $\omega$ between suspicious targets and standard vectors } \\
\hline & $\begin{array}{l}\mathrm{e}_{2} \\
\text { attack }\end{array}$ & $\begin{array}{l}\mathrm{e}_{3} \\
\text { reconni-ssance }\end{array}$ & $\begin{array}{l}\mathrm{e}_{9} \\
\text { cover }\end{array}$ & $\begin{array}{l}\mathrm{e}_{11} \\
\text { surveillance }\end{array}$ & $\begin{array}{l}\mathrm{e}_{1} \\
\text { other }\end{array}$ & $\begin{array}{l}\mathrm{e}_{13} \\
\text { other }\end{array}$ & $\begin{array}{l}\mathrm{e}_{14} \\
\text { other }\end{array}$ & $\begin{array}{l}\mathrm{e}_{15} \\
\text { other }\end{array}$ \\
\hline 30 & 0.069 & 1.610 & $\begin{array}{l}0.75 \\
1\end{array}$ & 0.223 & 1.216 & 1.086 & 2.673 & 0.107 \\
\hline 31 & 0.785 & 0.472 & $\begin{array}{l}2.03 \\
4\end{array}$ & 1.143 & 2.886 & 2.645 & 1.174 & 0.891 \\
\hline 36 & 0.168 & 1.251 & 1.01 & 0.414 & 1.570 & 1.412 & 2.193 & 0.261 \\
\hline 37 & 2.440 & 0.336 & $\begin{array}{l}4.80 \\
5\end{array}$ & 3.150 & 6.450 & 6.100 & 0.250 & 2.650 \\
\hline 39 & 0.818 & 0.487 & 2.10 & 1.172 & 2.945 & 2.710 & 1.119 & 0.910 \\
\hline 42 & 1.350 & 0.185 & $\begin{array}{l}3.05 \\
2\end{array}$ & 1.845 & 4.150 & 3.845 & 0.710 & 1.475 \\
\hline 51 & 0.412 & 0.860 & $\begin{array}{l}1.45 \\
3\end{array}$ & 0.725 & 2.130 & 1.940 & 1.665 & 0.529 \\
\hline 60 & 2.460 & 0.620 & $\begin{array}{l}4.85 \\
3\end{array}$ & 3.140 & 6.450 & 5.980 & 0.599 & 2.650 \\
\hline 72 & 0.327 & 2.320 & 0.38 & 0.090 & 0.742 & 0.640 & 3.645 & 0.225 \\
\hline 85 & 0.129 & 1.950 & $\begin{array}{l}0.52 \\
1\end{array}$ & 0.151 & 0.965 & 0.840 & 3.140 & 0.142 \\
\hline 91 & 1.730 & 0.195 & $\begin{array}{l}3.75 \\
1\end{array}$ & 2.320 & 4.980 & 4.680 & 0.570 & 1.920 \\
\hline 93 & 0.572 & 0.517 & 1.62 & 0.845 & 2.340 & 2.135 & 1.550 & 0.629 \\
\hline
\end{tabular}

The minimum deviation is marked (bold number) for each suspicious target, and the most probable intentions are shown in Table 11.

Table 11. Prediction of suspicious target intention

\begin{tabular}{c|l|c|l}
\hline $\begin{array}{l}\text { Suspicious } \\
\text { target ID }\end{array}$ & $\begin{array}{l}\text { Intension } \\
\text { prediction }\end{array}$ & $\begin{array}{l}\text { Suspicious } \\
\text { target ID }\end{array}$ & $\begin{array}{l}\text { Intension } \\
\text { prediction }\end{array}$ \\
\hline 30 & Attack & 51 & Attack \\
\hline 31 & Reconnaissance & 60 & Other \\
\hline 36 & Attack & 72 & Surveillance \\
\hline 37 & Other & 85 & Attack \\
\hline 39 & Reconnaissance & 91 & Reconnaissance \\
\hline 42 & Reconnaissance & 93 & Reconnaissance \\
\hline
\end{tabular}




\section{Validation of Suspicious Target Intention}

The deviation between suspicious targets are smaller than 0.3 for several standard vectors, which complicates rapid judgement of the target intention. Therefore, aspects of the targets motion curve were included for analysis, verifying the judgement accuracy of target intention based on vector deviation alone. Specifically:

(1) Target 30:

The most probable intention of suspicious target 30 is attack. The second most likely intention is other, corresponding to e15. The eigenvector is $(2300,216,138,311,7$, $3.5)$, and the azimuth angle, $\beta$; distance, $\mathrm{D}$; and course angle, $\theta$ are very close to standard vector e2 (attack class) $(2300,210,300,310,4.0,1.2)$, whereas the deviation for e15 is very large. Therefore, the intention of suspicious target 30 is judged to be attack.

(2) Target 31:

The most probable intention of suspicious target 31 is reconnaissance. The distance, D; horizontal velocity, v; and course angle, $\theta$ are very close to e 3 (reconnaissance class).

(3) Target 36:

The most probable intention of suspicious target 36 is attack. The second closest standard vector is other (e15). However, each data value of the eigenvector is closer to the corresponding value of standard vector e2.

(4) Target 37:

The most probable intention of suspicious target 37 is other, corresponding to the standard vector e1. The second most likely intention is reconnaissance, but the azimuth angle is too small and the vision is narrow. Hence the vector deviation from reconnaissance is large and excluded ${ }^{[9]}$, and the intention determined to be other.

(5) Target 39:

The most probable intention of suspicious target 39 is reconnaissance. The second most likely intention is attack. However, the velocity is too small, i.e., target 39 does not have attack velocity, and the vector deviation from standard vector e2 (attack class) is much larger than the minimum.

(6) Target 39:

The intention of suspicious target 39 is reconnaissance. The eigenvector agrees well with standard vector e3 (reconnaissance class), and all other deviations for the remaining cases are large.

(7) Target 51:

The most probable intention of suspicious target 51 is attack. The second most likely intention is other (e15). However, each parameter of the suspicious target is very close to the corresponding parameter of e2, and Fig. 2 shows that the distance to the radar is only $168 \mathrm{~km}$, with flight direction and velocity very close to the attack case. Therefore, the intention of suspicious target 51 is assessed to be attack.

(8) Target 60

The probables intention of suspicious target 60 is reconnaissance and other. However, Fig. 2 shows suspicious target 60 is receding from the radar, which argues a nonmilitary intention. The azimuth angle is also poor for reconnaissance. Accordingly, the intention of suspicious target 60 is determined to be other.

(9) Target 72: 
The intention of suspicious target 72 is surveillance. The eigenvector agrees well with e11 (surveillance class), and Fig. 2 shows that the distance between the suspicious target and the radar is maintained.

\section{(10) Target 85:}

The most probable intention of suspicious target 85 is attack or other (e15). The attack angle is excellent ${ }^{[10]}$, distance to radar is smal, and flight velocity is very large, close to standard vector e15. Therefore, the intention of suspicious target 85 is confirmed as attack.

(11) Target 91:

The intention of the suspicious target with ID 91 is reconnaissance. The eigenvector is very close to the standard vector e3 (reconnaissance class), and Fig. 2 shows constant distance to the radar. Therefore, the intention assessed to be reconnaissance.

\section{(12) Target 93:}

The most probable intention of suspicious target 93 is reconnaissance, and the second is attack. Figure 5 shows the distance to the radar decreases in the first half then is maintained in the second half of the time. Additionally, the course angle is strongly characteristic of reconnaissance [11]. The vector deviation with standard vector e2 (attack class) is large. Therefore, the intention of suspicious target 93 is determined to be attack.

\section{Conclusions}

Using the proposed vector deviation, the intention of suspicious targets were assessed, and validated. Vector deviation was confirmed as an effective prediction mechanism for target intention.

When the vector deviation calculated by the target vector and the standard vector with different intentions were very close, there existed partial inference for the rapid judgement of the target intention. This was due to the fact that the effect of each composition element in each target eigenvector on the vector was different. In the practical situation, the weighting of each element should be given according to its significance in the vector $[12,13]$. For example, the weightings of azimuth angle $\beta$, distance $\mathrm{D}$, course angle $\theta$ and velocity $\mathrm{v}$ for the judgement of the suspicious target intention were obviously higher than that of height $\mathrm{H}$ and radar cross section $\sigma$. Moreover, the elements influenced each other. It can be assumed that the target eigenvector after weighting revising $\mathrm{e}=\left(\eta_{\beta^{*}} \beta, \eta_{\mathrm{D}^{*}} \mathrm{D}, \eta_{\mathrm{v}^{*} \mathrm{~V}}, \eta_{\theta^{*}} \theta, \eta_{\mathrm{H}^{*}} \mathrm{H}, \eta_{\sigma^{*}} \sigma\right)$, where $\eta_{\beta}$, $\eta_{\mathrm{D}}, \eta_{\mathrm{v}}, \eta_{\theta}, \eta_{\mathrm{H}}$ and $\eta_{\sigma}$ were the weightings of the corresponding elements [14]. The weightings were needed to determine according to large amounts of data [15]. Then the eigenvector after being given weighting was applied to the calculation of the deviation degree. As a result, the more accurate vector deviation can be obtained.

The assessment of suspicious target intention using vector deviation is rapid and precise. The problem of weighting of each element in the target eigenvector would be referred to in the future study.

\section{Acknowledgements}

At the point of finishing this paper, I would like to express my sincere thanks to Organizing Committee of National Post-Graduate Mathematic Contest in Modeling and Taiyuan University Of Technology Graduate School. 


\section{Reference}

[1]Bingjian Li, Xiaoqiang Wu, Features and Development Orientation of The Modern Radar Reconnaissance Technologies, Shipboard Electronic Counter Measure.30(2007)19-21.

[2]Jianyu Yang, Development Laws and Macro Trends Analysis of Radar Technology, Journal of Radar.1(2012)19-27.

[3]Mingyan Ruan, The Vector Application And Teaching Study of Mathematics, Huazhong Normal University,Wuhan,2007.

[4]Sandro Caparrini, The Discovery of the Vector Representation of Moments and Angular Velocity, Archive for History of Exact Sciences .56 (2002)151-181.

[5]Jun Wu, Beauty of Mathematics, People's Posts and Telecommunications Press, Beijing, 2012.

[6]Xiangqiang Kong, Exploring The Application of MATLAB Software in Space Analytical geometry teaching,Computer Applications and Software,29(2012)12-17.

[7]Ping Zhang, MATLAB Basic and Applied, Beijing University of Aeronautics and Astronautics Press, Beijing, 2007.

[8]Yuan Song, Xinhua Zhang, Weidong Guo, Hierarchical Inference Frame and Realization of Air Target Tactical Intention, Information Command Control System and Simulation Technology,27(2005)63-66.

[9]Jiyan Du, Lianfei Duan, Guoman Huang, Principle of Target Location for UAV TV Reconnaissance, Press of University of Science and Technology of China,Hefei,2013.

[10]Rui Wang, The Combat Effectiveness Evaluation of Advanced Battle Plane, Northwestern Polytechnical University,Xian,2007.

[11]Bo Sun, Research of Control System of Autopilot for Unmanned Aerial Vehicle, Harbin Engineering University,Harbin,2012.

[12]Shucai Tai, Yunyu Sun,Juanjuan He, Applied mathematical statistics, Wuhan University Press, Wuhan,2005.

[13]Zhongbi Li, Xiaobai Li, The Basis and Dimension of n-dimensional Vector Space and Intersection Space, Journal of Chongqing Normal University,19(2002)80-84.

[14]Glose Daniel,Kennel Ralph, Continuous Space Vector Modulation for Symmetrical Six-Phase Drives, IEEE Transactions on Power Electronics.31(2016)3837-3848.

[15]Xiuying Cao, Jingguo Liang, The Method of Ascertaining Attribute Weight Based on Rough Sets Theory, Chinese Journal of Management Science.10(2002)98-100. 